\title{
Synthesis and Biological Evaluation of Epothilone A Dimeric Compounds
}

\section{Daniele Passarella, ${ }^{\star} a$ Daniela Comi, ${ }^{a}$ Graziella Cappelletti, ${ }^{b}$ Daniele Cartelli, ${ }^{b}$ Juerg Gertsch, ${ }^{c}$ Ana R. Quesada, ${ }^{1, d}$ Jurgen Borlak, ${ }^{1, e}$ Karl-Heinz Altmann ${ }^{1, c}$}

${ }^{a}$ Dipartimento di Chimica Organica e Industriale, Università degli Studi di Milano, Via Venezian 21, - ${ }^{b}$ Dipartimento di Biologia, Università degli Studi di Milano, Via Celoria 26, 20133 Milano (Italy) - ${ }^{c}$ ETH Zurich, Department of Chemistry and Applied Biosciences, Institute of Pharmaceutical Sciences, HCI H 405, 8093 Zurich (Switzerland) - ${ }^{d}$ Departamento de Biología Molecular y Bioquímica, Universidad de Málaga, E-29071 Malaga (Spain) - ${ }^{e}$ Fraunhofer Institute of Toxicology and Experimental Medicine Medical School of Hannover, NikolaiFuchs-Str 1, 30625 Hannover (Germany)

Dipartimento di Chimica Organica e Industriale, Università degli Studi di Milano, Via Venezian 21, 20133 Milano, Italy, Tel. 02.50314081, FAX 02.50314078. E.mail: Daniele.Passarella@unimi.it

${ }^{[1]}$ contributed equally as senior authors

\begin{abstract}
The preparation and biological evaluation of a novel series of dimeric epothilone A derivatives (1 - 6) are described. Two types of diacyl spacers were introduced to establish the various dimeric epothilone A constructs. The effect of these compounds on tubulin polymerization and their cytotoxicity against four different cancer cell lines are reported. Several of the newly synthesized compounds inhibit endothelial cell differentiation and endothelial cell migration that are key steps of the angiogenic process.
\end{abstract}

Keywords: Epothilone A · tubulin polymerization · inhibition of angiogenesis

\section{Introduction}

Substances that are able to interfere with the tubulin-microtubule system, either through inhibition of tubulin polymerization or through microtubule stabilization, are important anticancer agents. Prominent examples are the vinca alkaloids (which act as tubulin polymerization inhibitors) or the diterpenoid alkaloid taxol and its semi-synthetic derivative docetaxel (which are microtubule stabilizers), all of which are indispensable components of modern cancer chemotherapy regimens. ${ }^{1}$ The most recent addition to the clinical arsenal of tubulin inhibitors is the lactam analog of the bacterial natural product epothilone $\mathrm{B}^{2}$ (Epo B, Figure 1), which has been approved for clinical use by the US FDA in late 2007 under the trade name Ixempra ${ }^{\circledR}$ (generic name: ixabepilone). ${ }^{3}$ In 
addition to their growth-inhibitory and apoptosis-inducing activity, some tubulin inhibitors have recently been shown to target the vascular system of tumors, ${ }^{4}$ thereby causing vasculature recession (induction of apoptosis by a classical anti-angiogenic mechanism) or vasculature "normalization”. 5 Vasculature normalization facilitates and enhances tumor cell penetration by anticancer drugs, thus offering the opportunity for extensive tumor kill by appropriate drug combinations. As a consequence, the identification of new tubulin modulators ${ }^{6}$ remains an important challenge in anticancer drug discovery and the use of well known tubulin-interacting agents as scaffolds is a represents reasonable approach towards this goal. In this context, we have been intrigued by a dimeric epothilone analog (Epo-Dim, Figure 1) that has been reported by Nicolaou and co-workers ${ }^{7}$ several years ago as an undesired side product in the course of the target-oriented synthesis of a series of side chain-modified epothilones. Although no specific data were provided for this analog, ${ }^{7}$ the compound is stated to exhibit remarkable tubulin-polymerizing and antiproliferative activity. Building on these earlier findings we have now synthesized a set of a epothilone dimers that are more readily accessible than Epo-Dim and we have investigated the in vitro biological properties of these new epothilone derivatives (Scheme 1$)^{8}{ }^{8}$
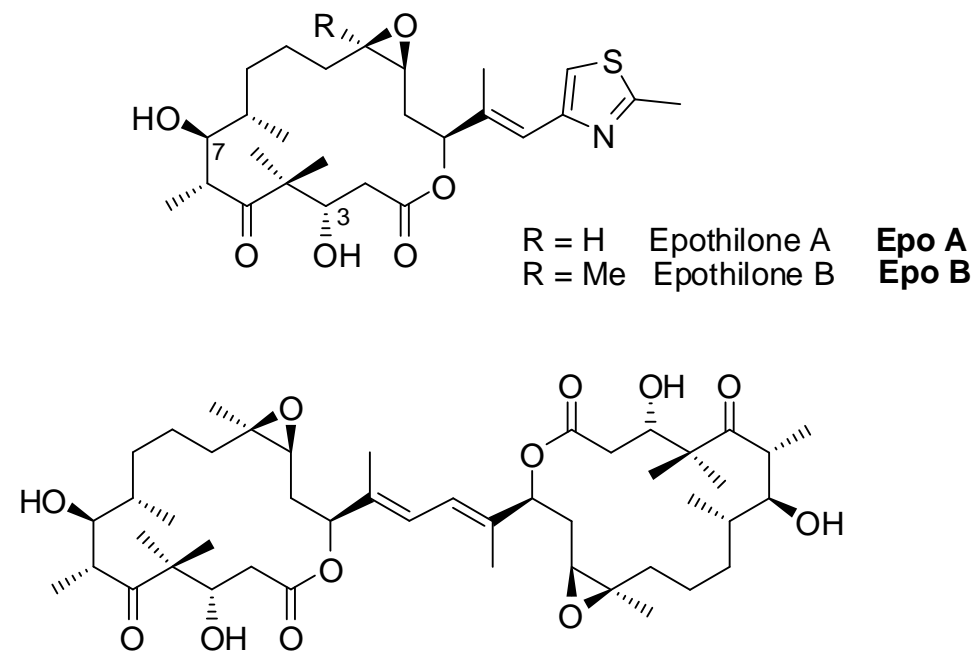

K.C. Nicolaou dimeric compound Epo-Dim

Figure 1: Epothilones A and B. Structure of the dimeric epothilone analog Epo-Dim described by K.C. Nicolaou (Ref. 7). 


\section{Results and Discussion}

\subsection{Chemistry}

The presence of two hydroxyl groups in the epothilone macrocycle offered an opportunity for the direct introduction of a spacer moiety between two epothilone monomers. Assuming the OH-group on C3 to be sterically more hindered, we felt that the acylation of C7-OH should be feasible with reasonable selectivity and thus provide C7 linked dimers as the major prodcuts of the reaction of Epo A with activated dicarboxylic acids. ${ }^{9}$ While being aware of the potential in vivo lability of the ester function our initial objective was to prepare a number of prototypical dimers in the simplest way possible and to evaluate these compounds in tubulin polymerisation assays. Thus reaction of Epo A with six different dicarboxylic acids in the presence of DCC and DMAP (Scheme 1) gave the corresponding products $(\mathbf{1}-\mathbf{6})$ with excellent regioselectivity. The latter compensated at least partially, for the low to moderate yields obtained in the esterification reactions (25 - $40 \%)$. Esterification of the C7-OH group in the derivatives isolated (rather than $\mathrm{C3}-\mathrm{OH}$ ) was demonstrated by NMR spectroscopy. The spectra of $\mathbf{1}$ - $\mathbf{6}$ exhibit a single set of signals for the epothilone unit with chemical shifts that are similar to those in the spectrum of Epo A, except for the signal for H7, which is down-shifted to $\delta 5.37$ - 5.44 (Epo A $\delta$ 3.80). All compounds appeared sufficiently stable chemically under the conditions of the biological experiments.

Epo A<smiles>[X][X]C(=O)C(=O)O</smiles>
DCC, DMPA 6-8 h

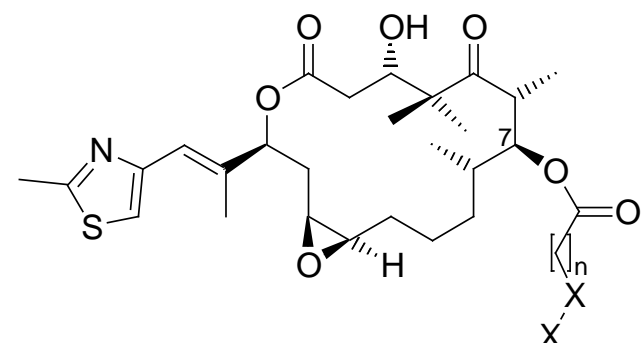

$1-6$

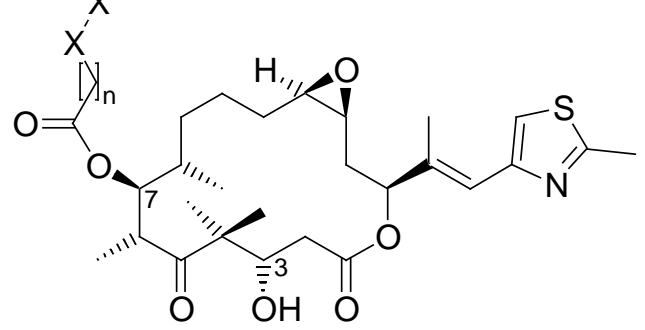




\begin{tabular}{|c|cccccc|}
\hline Compound & $\mathbf{1}$ & $\mathbf{2}$ & $\mathbf{3}$ & $\mathbf{4}$ & $\mathbf{5}$ & $\mathbf{6}$ \\
\hline$n$ & 1 & 2 & 3 & 1 & 2 & 3 \\
$x$ & $\mathrm{~S}$ & $\mathrm{~S}$ & $\mathrm{~S}$ & $\mathrm{CH}_{2}$ & $\mathrm{CH}_{2}$ & $\mathrm{CH}_{2}$ \\
\hline
\end{tabular}

Scheme 1: General preparation of derivatives $\mathbf{1 - 6 .}$

\subsection{Influence on Tubulin Polymerization}

In order to assess the interaction of compounds $\mathbf{1} \mathbf{- 6}$ with the tubulin/microtubule system, they were tested in an in vitro tubulin polymerization assay at fixed concentrations of 5 and $10 \mu \mathrm{M}$. As illustrated by the data in Table 1, Epo A at these concentrations induces almost complete assembly of soluble tubulin into microtubule polymers. In contrast, no physically meaningful tubulin assembly could be detected for any of the dimeric analogs $\mathbf{1}-\mathbf{6}$ under the same experimental conditions. While it cannot be excluded that tubulin polymerization might be detectable under a different set of experimental conditions, it is clear from the data in Table 1 that dimers $\mathbf{1}-\mathbf{6}$ are substantially less potent inducers of tubulin polymerization than Epo A in vitro (if any).

Given the chemical stability of all the dimeric compounds $\mathbf{1}$ - $\mathbf{6}$ under the conditions of a cell culture assay and in light of a noticeably improved solubility (compared with Epo A) the antiproliferative activity of these compounds was evaluated against a series of cancer cells.

\begin{tabular}{|c|cc|ccccc|c|c|c|}
\hline Compound & \% Tubulin Polym. ${ }^{[a]}$ & A-549 & MCF-7 & HT-1080 & $\beta D 10^{[b]}$ & BAE & Tubes & Migration \\
\hline \multicolumn{7}{|c|}{$(10 \mu \mathrm{M})$} & $(5 \mu \mathrm{M})$ & \multicolumn{4}{c|}{ MIC $C^{[d]}$} \\
\hline Epo A & $99.8 \pm 0.4$ & $92.2 \pm 2.3$ & $2.6 \pm 0.23$ & $2.5 \pm 0.29$ & $1.3 \pm 0.5$ & 70 & $0.7 \pm 0.4$ & 5 & 5 \\
$\mathbf{1}$ & $1 \pm 0.5$ & $0.3 \pm 0.4$ & $319 \pm 21$ & $357 \pm 44$ & $290 \pm 98$ & 1000 & $29 \pm 3$ & 100 & 50 \\
$\mathbf{2}$ & $0.2 \pm 0.4$ & $0 \pm 0.3$ & $367 \pm 18$ & $379 \pm 20$ & $43 \pm 18$ & 3.3 & $111 \pm 73$ & 50 & 100 \\
$\mathbf{3}$ & $0.2 \pm 0.6$ & $0.5 \pm 0.7$ & $383 \pm 30$ & $432 \pm 17$ & $71 \pm 15$ & 2000 & $133 \pm 30$ & 100 & $100^{*}$ \\
$\mathbf{4}$ & $0.7 \pm 0.4$ & $0.2 \pm 0.4$ & $405 \pm 27$ & $412 \pm 18$ & $69 \pm 22$ & 70500 & $40 \pm 10$ & 100 & 100 \\
$\mathbf{5}$ & $0.3 \pm 0.5$ & $0.7 \pm 0.5$ & $316 \pm 17$ & $321 \pm 28$ & $17 \pm 13$ & 2500 & $20 \pm 13$ & 100 & 100 \\
$\mathbf{6}$ & $0.6 \pm 0.5$ & $0.1 \pm 0.7$ & $956 \pm 43$ & $1033 \pm 39$ & $13 \pm 3$ & 5020 & $39 \pm 8$ & 50 & $100^{*}$ \\
\hline
\end{tabular}

Table 1. Biological evaluation of compounds $1-$ 6. [a] Polymerization was monitored at a fixed compound concentratin of 5 or $10 \mu \mathrm{M}$ by following the increase in absorption at $340 \mathrm{~nm}$; [b] Cells from lung tumors of double cmyc and c-raf transgenic mice; [c] Compound concentration, in $\mathrm{nM}$, that causes 50\% inhibition of the control cell growth, data are means \pm S.D. of at least three independent experiments with triplicate samples each; [d] Minimal inhibitory concentration of compounds 1-6 (MIC) on BAE (bovine aortic endothelial) cell differentiation (Tube formation) or migration. The different assay was carried out in the presence of different concentrations of Epo A and dimers 1-6 as described in Experimental Section. Migration assay was carried out in the presence of different concentrations of epothilone A and related compounds as described in Experimental Section. 


\subsection{Cytotoxicity}

The anticancer activities of the new compounds were examined in three different human cancer cell lines, namely A549 (lung cancer), MCF7 (breast cancer), and HT1080 (fibrosarcoma) and in one transformed mouse cell line that is derived from lung tumors of double c-myc and c-raf transgenic mice $(\beta \mathrm{D} 10)^{10}$ (Table 1$)$. With one exception all compounds investigated were found to be less active than the parent compound Epo A, thus reflecting the reduced ability of the dimeric analogs to induce tubulin polymerization in vitro. It remains to be determined, whether the observed cellular activity is in fact associated with the dimers as such or whether it may be a consequence of (partial) enzyme-mediated ester hydrolysis (either in the cell culture medium or inside the cells) and the ensuing release of free Epo A. As an exception, the stem cell line $\beta \mathrm{D} 10$ appeared to be more sensitive to 2 than to Epo A. The reason for this behavior are unclear at this point.

\subsection{Anti-angiogenic activity}

Angiogenesis involves local proliferation of endothelial cells. ${ }^{11}$ Therefore, we also investigated the ability of Epo A and dimers 1-6 to inhibit the growth of endothelial cells. As illustrated by the IC $_{50}$ values shown in Table 1, the dimeric epothilone analogs inhibit endothelial cell growth at submicromolar concentrations. But they are clearly less potent than the parent compound Epo A. Comparison of these results with those obtained with tumor cells (previous paragraph) demonstrates that the inhibitory effect is not endothelial cell specific in the case of compounds 2-6 and Epo A. The data suggest, however, that compound $\mathbf{1}$ could present a higher cell growth inhibitory activity for endothelial cells when compared to that on tumor cells.

The final event during angiogenesis is the organisation of endothelial cells into a three-dimensional network of tubes. In vitro, endothelial cells plated on Matrigel align themselves forming cords, already evident a few hours after plating. Figure $2 \mathrm{~b}$ shows that 5 nM Epo A was able to completely inhibit the BAE cell alignment and cord formation. Dimerization of Epo A decreases the endothelial morphogenesis inhibitory activity. An inhibition of endothelial morphogenesis on Matrigel was obtained with $50 \mathrm{nM}$ compound 6, or with $100 \mathrm{nM}$ compounds 1-5 (Figure 2c-h). 
Angiogenesis involves the acquisition by endothelial cells of the capability to migrate through the extracellular matrix, degrade the basement membrane and, in general, to remodel the extracellular matrix. Our results, presented in Figure 3a, show that 5 nM Epo A produced a significant inhibition of the migratory capability of BAE cells. As shown in Figure 3b-h, a significant effect on BAE cells migration was observed in the presence of compound $\mathbf{1}(50 \mathrm{nM})$ or compounds 2-6 (100 nM).
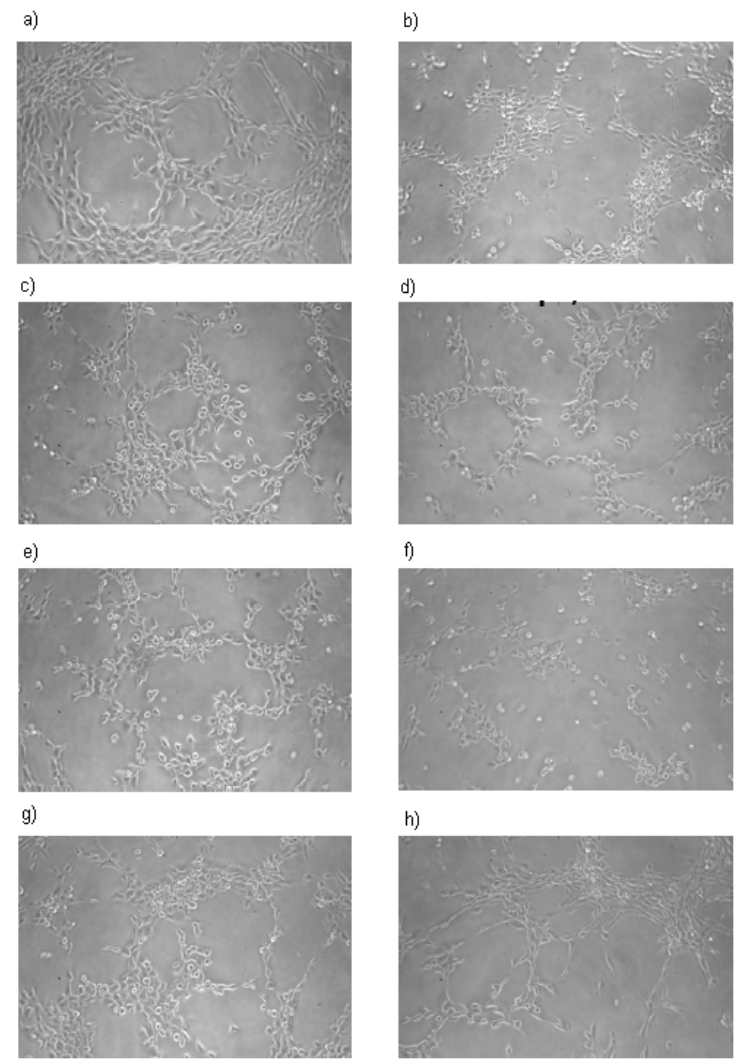

Figure 2. Effects of Epo A and dimeric derivatives on endothelial cells: a) control; b) Epo A, 5 nM; c) 1, 100 nM; d) 2 $100 \mathrm{nM}$; e) 3, $100 \mathrm{nM}$;) 4, $100 \mathrm{nM}$; g) 5, $100 \mathrm{nM}$; h) 6, $50 \mathrm{nM}$.

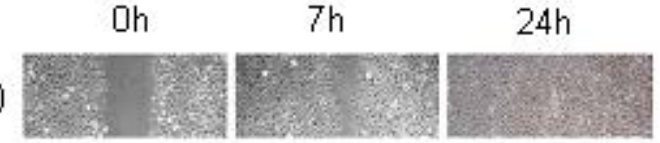

b)

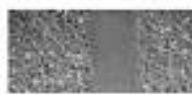

c)

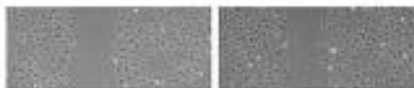

d)

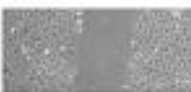

e)
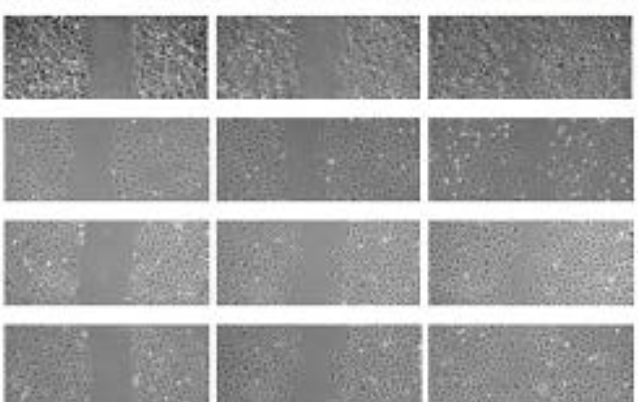

f)
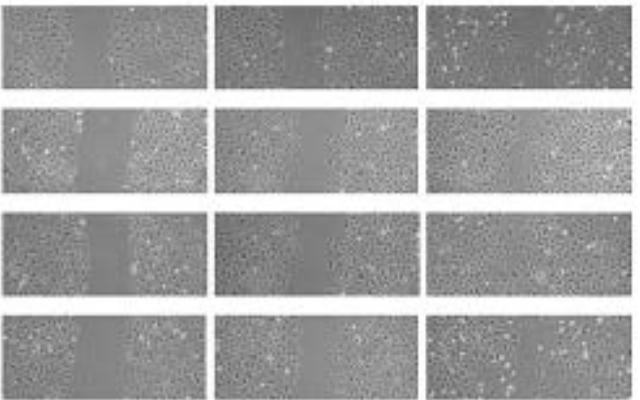

g)
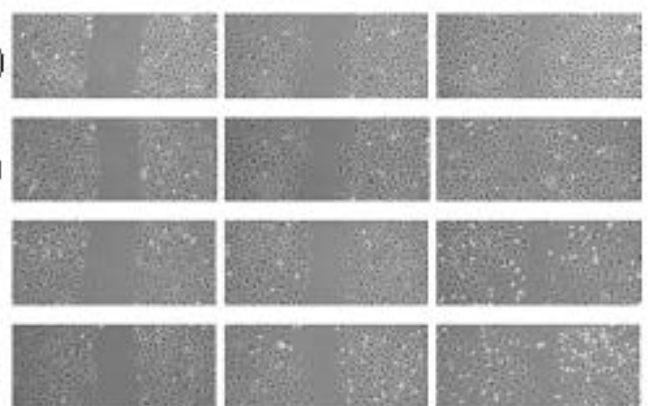

h)

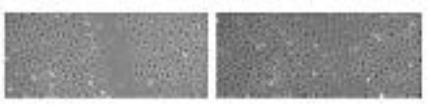


Figure 3. Effect of Epo A and dimeric derivatives on endothelial cell migration. a) control; b) Epo A, 5 nM; c) 1, 50 nM; d) 2, 100 nM; e) 3, 100 nM; f) 4, $100 \mathrm{nM;} \mathrm{g)} \mathrm{5,} 100 \mathrm{nM;} \mathrm{h)} \mathrm{6,} 100 \mathrm{nM}$.

\section{Conclusions}

Our results indicate that several Epo A related dimeric compounds inhibit endothelial cell differentiation and endothelial cell migration, which are key steps of the angiogenic process, at nontoxic concentrations. The discovery that these compounds interfere with several functions of activated endothelial cells suggests that they could be of interest for the development of new agents for the treatment of angiogenesis-related diseases.

\section{Experimental Section}

General: Thin-layer chromatography (TLC) was performed on Merck precoated 60F254 plates. Reactions were monitored by TLC on silica gel, with detection by UV light (254 nm) or by charring with sulfuric acid. Flash chromatography was performed using Silica gel (240-400 mesh, Merck). ${ }^{1} \mathrm{H}$ NMR spectra were recorded with Brucker 200, 300 and $400 \mathrm{MHz}$ spectrometers using chloroform-d $\left(\mathrm{CDCl}_{3}\right)$ and methanol- $\mathrm{d}_{4}\left(\mathrm{CD}_{3} \mathrm{OD}\right)$. Chemical shifts are reported in parts per million (ס) downfield from tetramethylsilane (TMS) as internal standard. EI mass spectra were recorded at an ionizing voltage of $6 \mathrm{Kev}$ on a VG 70-70 EQ. ESI mass spectra were recorded on FT-ICR APEX $^{\mathrm{II}}$ (Bruker Daltonics). All reactions were carried out in dry solvents.

Cell culture media were purchased from Gibco (Grand Island, NY, USA) and Biowhittaker

(Walkersville, MD, USA). Fetal bovine serum (FBS) was a product of Harlan-Seralab (Belton, U.K.). Matrigel was purchased from Becton Dickinson (Bedford, MA, USA). Supplements and other 
chemicals not listed in this section were obtained from Sigma Chemicals Co. (St. Louis, Mo., USA). Plastics for cell culture were supplied by NUNC (Roskilde, Denmark). Epothilone A and related compounds (figure 1) were dissolved in dimethylsufoxide (DMSO) at a concentration of $10 \mathrm{mM}$ and stored at $-20^{\circ} \mathrm{C}$ until use. Bovine aortic endothelial (BAE) cells were obtained by collagenase disgestion and maintained in Dulbecco's modified Eagle’s medium containing glucose (1g/L), glutamine (2mM), penicillin (50 IU/mL), streptomycin $(50 \mu \mathrm{g} / \mathrm{mL})$, and amphoterycin $(1.25 \mu \mathrm{g} / \mathrm{mL})$ (DMEM) supplemented with 10\% FBS (DMEM/10\%FBS). Human fibrosarcoma HT1080 cells were obtained from the ATCC and maintained in DMEM containing glucose (4.5 g/L), glutamine (2mM), penicillin (50 IU/mL), streptomycin (50 $\mu \mathrm{g} / \mathrm{mL})$, and amphoterycin $(1.25 \mu \mathrm{g} / \mathrm{mL})$ supplemented with $10 \%$ FBS.

General procedure for preparation of compounds 1 - 6: DMAP (0.05 mmol), epothilone (0.1 mmol) and DCC $(0.15 \mathrm{mmol})$ were added to a solution of diacid $(0.05 \mathrm{mmol})$ in $\mathrm{CH}_{2} \mathrm{Cl}_{2}$ dry at room temperature. The mixture was stirred for 6-8 hours then concentrated under reduced pressure. The crude residue was purified by flash chromatography (silica gel, AcOEt/Hexane 3:2) to give the dimeric compounds. Yields: 25 - $40 \%$.

Epothilone A: ${ }^{1} \mathrm{H}$ NMR (400 MHz, $\left.\mathrm{CDCl}_{3}\right) \delta 6.99(1 \mathrm{H}, \mathrm{s}), 6.61(1 \mathrm{H}, \mathrm{s}), 5.44(1 \mathrm{H}, \mathrm{dd}, J=2.5,8.7$ Hz), 4.21-4.19 (1H, m), 3.95 (1H, bs), 3.80 (1H, t, $J=4.3$ Hz), 3.50 (1H, br s), 3.27-3.21 (1H, m), 3.06-3.02 (1H, m), 2.94-2.90 (1H, m), $2.71(3 \mathrm{H}, \mathrm{s}), 2.66(1 \mathrm{H}$, br s), $2.55(1 \mathrm{H}, \mathrm{dd}, J=14.5,10.5$ Hz), 2.42 (1H, dd, $J=14.5,3.2 \mathrm{~Hz}), 2.17-2.12(1 \mathrm{H}, \mathrm{m}), 2.11(3 \mathrm{H}, \mathrm{s}), 1.93-1.85(1 \mathrm{H}, \mathrm{td}, J=15.1$, 8.4 Hz), 1.78-1.72 (2H, m), 1.57-1.41 (5H, m), 1.38 (3H, s), 1.18 (3H, d, J = 6.8 Hz), 1.11 (3H, s), $1.01(3 \mathrm{H}, \mathrm{d}, J=7.0 \mathrm{~Hz}) ;{ }^{13} \mathrm{C}$ NMR (100.6 MHz, $\left.\mathrm{CDCl}_{3}\right) \delta 218.8$ (1C), 171.1 (1C), 165.8 (1C), 152.4 (1C), 138.2 (1C), 120.4 (1C), 116.8 (1C), 77.2 (1C), 75.2 (1C), 73.8 (1C), 58.0 (1C), 55.2 
(1C), 44.0 (1C), 39.6 (1C), 36.9 (1C), 32.2 (1C), 31.2 (1C), 27.8 (1C), 24.1 (1C), 22.2 (1C), 21.0 (1C), 19.6 (1C), 17.7 (1C), 16.3 (1C), 14.6 (1C).

$1:{ }^{1} \mathrm{H}$ NMR (400 MHz, $\left.\mathrm{CDCl}_{3}\right) \delta 7.01$ (2H, s), 6.67 (2H, s), 5.52 (2H, dd, $\left.J=6.5,2.1 \mathrm{~Hz}\right), 5.44$ (2H, dd, $J=1.7,8.9 \mathrm{~Hz}), 4.17-4.12(2 \mathrm{H}, \mathrm{m}), 3.5-3.4$ (2H, dd, $J=9.83,6.99 \mathrm{~Hz}), 3.12-3.05(2 \mathrm{H}, \mathrm{m})$, 2.95-2.88 (2H, m), 2.73 (6H, s), 2.60-2.55 (4H, m), 2.15 (6H, s), 2.13-2.10 (2H, m), 2.00-1.98 (2H, m), 1.82-1.79 (2H, m), 1.66-1.55 (8H, m), 1.40 (8H, s), 1.30-1.26 (6H, s), 1.13 (6H, s), 1.10 (6H, d, $J=6.8 \mathrm{~Hz}), 0.96(6 \mathrm{H}, \mathrm{d}, J=6.8 \mathrm{~Hz}) ;{ }^{13} \mathrm{C} \mathrm{NMR}\left(100.6 \mathrm{MHz}, \mathrm{CDCl}_{3}\right) \delta 216.1(2 \mathrm{C}), 170.3(2 \mathrm{C})$, 168.7 (2C), 164.9 (2C), 152.1 (2C), 136.8 (2C), 119.8 (2C), 116.3 (2C), 80.2 (2C), 76.0 (2C), 73.9 (2C), 57.3 (2C), 53.7 (2C), 52.3 (2C), 43.8 (2C), 38.4 (2C), 34.4 (2C), 30.8 (2C), 29.2 (4C), 26.2 (2C), 24.1 (2C) , 22.2 (2C), 20.8 (2C), 18.9 (2C), 17.4 (2C), 16.6 (2C), 15.5 (2C); Anal. Calcd. for $\mathrm{C}_{56} \mathrm{H}_{80} \mathrm{~N}_{2} \mathrm{O}_{14} \mathrm{~S}_{4}$ : C , 59.34; H, 7.11; N, 2.47. Found: C , 59.38; H, 7.15; N, $2.43[\alpha]_{\mathrm{D}}{ }^{25}=-2.2$ (c 0.85, $\mathrm{CHCl}_{3}$ ); HRESI positive MS: Anal. Calcd. for $\mathrm{C}_{56} \mathrm{H}_{80} \mathrm{~N}_{2} \mathrm{O}_{14} \mathrm{~S}_{4} \mathrm{Na}^{+}, 1155.43846$; found 1155.43862.

$2:{ }^{1} \mathrm{H}$ NMR (400 MHz, $\left.\mathrm{CDCl}_{3}\right) \delta 7.00$ (2H, s), 6.64 (2H, s), 5.50 (2H, br. d, $\left.J=4.7 \mathrm{~Hz}\right), 5.41(2 \mathrm{H}$, d, $J=9.1 \mathrm{~Hz}), 4.16-4.11(2 \mathrm{H}, \mathrm{m}), 3.44-3.36(2 \mathrm{H}, \mathrm{m}), 3.16-3.06(2 \mathrm{H}, \mathrm{m}), 2.98(4 \mathrm{H}, \mathrm{t}, J=7.1 \mathrm{~Hz})$, 2.93-2.90 (2H, m), 2.80 (8H, t, $J=7.0 \mathrm{~Hz}), 2.72$ (6H, s), 2.60-2.51 (4H, m), 2.13 (6H, s), 2.07-1.94 (4H, m), 1.80-1.71 (2H, m), 1.68-1.53 (8H, m), 1.39 (6H, s), 1.40-1.35 (2H, m), 1.29-1.24 (2H, m), $1.12(6 \mathrm{H}, \mathrm{s}), 1.09(6 \mathrm{H}, \mathrm{d}, J=6.7 \mathrm{~Hz}), 0.95(6 \mathrm{H}, \mathrm{d}, J=6.7 \mathrm{~Hz}) .{ }^{13} \mathrm{C}$ NMR $\left(100.6 \mathrm{MHz}, \mathrm{CDCl}_{3}\right) \delta$ 217.2 (2C), 171.8 (2C), 171.1 (2C), 165.5 (2C), 152.8 (2C), 137.2 (2C), 120.7 (2C), 117.0 (2C), 79.7 (2C), 76.7 (2C), 74.7 (2C), 58.0 (2C), 54.5 (2C), 53.0 (2C), 44.4 (2C), 39.0 (2C), 35.0 (2C), 34.8 (2C), 34.0 (2C), 32.0 (2C), 29.9 (2C) 26.9 (2C), 24.7 (2C), 22.9 (2C), 21.0 (2C), 19.7 (2C), 18.2 (2C), 16.3 (2C), 16.2 (2C); Anal. Calcd. for $\mathrm{C}_{58} \mathrm{H}_{84} \mathrm{~N}_{2} \mathrm{O}_{14} \mathrm{~S}_{4}$ : C, 59.97; H, 7.29; N, 2.41. Found: C, 59.99; H, 7.31; N, $2.43[\alpha]_{\mathrm{D}}^{25}=-2.96$ (c 0.455, $\mathrm{CHCl}_{3}$ ); HRESI positive MS: Anal. Calcd. for $\mathrm{C}_{58} \mathrm{H}_{84} \mathrm{~N}_{2} \mathrm{O}_{14} \mathrm{~S}_{4} \mathrm{Na}^{+}$, 1183.46976; found 1183.46837 . 
$3:{ }^{1} \mathrm{H}$ NMR (400 MHz, $\left.\mathrm{CDCl}_{3}\right) \delta 7.05$ (2H, s), 6.63 (2H, s), $5.51(2 \mathrm{H}, \mathrm{dd}, J=2.3,6.8 \mathrm{H}), 5.38(2 \mathrm{H}$, dd, $J=1.9,8.7 \mathrm{~Hz}), 4.14-4.11$ (2H, m), 3.42-3.38 (2H, m), 3.10-3.06 (2H, m), 2.93-2.90 (2H, m), $2.78(4 \mathrm{H}, \mathrm{t}, J=7.0 \mathrm{~Hz}), 2.72(6 \mathrm{H}, \mathrm{s}), 2.61-2.54(4 \mathrm{H}, \mathrm{m}), 2.50$ (4H, t, $J=7.3 \mathrm{~Hz}), 2.15$ (6H, s), 2.15$2.10(2 \mathrm{H}, \mathrm{m}), 2.06$ (4H, t, $J=7.1 \mathrm{~Hz}), 1.99-1.95$ (2H, m), 1.80-1.76 (2H, m), 1.65-1.55 (8H, m), 1.38 (6H, s), 1.33-1.18 (4H, m), 1.13 (6H, s), 1.08 (6H, d, $J=6.9 \mathrm{~Hz}), 0.94(6 \mathrm{H}, \mathrm{d}, J=6.8 \mathrm{~Hz}) ;{ }^{13} \mathrm{C}$ NMR (100.6 MHz, $\mathrm{CDCl}_{3}$ ) $\delta 216.2$ (2C), 172.3 (2C), 170.4 (2C), 164.3 (2C), 152.2 (2C),136.6 (2C), 120.0 (2C), 116.3 (2C), 78.4 (2C), 76.1 (2C), 73.9 (2C), 57.3 (2C), 53.8 (2C), 52.3 (2C), 43.7 (2C), 38.5 (2C), 38.0 (2C), 34.5 (2C), 32.6 (2C), 31.8 (2C), 29.4 (2C), 26.3 (2C), 24.4 (2C), 24.1 (2C), 22.1 (2C), 20.4 (2C), 19.0 (2C), 17.5 (2C), 15.5 (2C), 15.3 (2C); Anal. Calcd. for $\mathrm{C}_{60} \mathrm{H}_{88} \mathrm{~N}_{2} \mathrm{O}_{14} \mathrm{~S}_{4}$ : C, 60.58; H, 7.46; N, 2.35. Found: C, 60.61; H, 7.48; N, 2.38. $[\alpha]_{D}^{25}=-3.8(\mathrm{c}=$ 0.554, $\mathrm{CHCl}_{3}$ ); HRESI positive MS: Anal. Calcd. for $\mathrm{C}_{60} \mathrm{H}_{88} \mathrm{~N}_{2} \mathrm{O}_{14} \mathrm{~S}_{4} \mathrm{Na}^{+}$, 1211.50106; found 1211.50400.

$4:{ }^{1} \mathrm{H}$ NMR (400 MHz, $\left.\mathrm{CDCl}_{3}\right) \delta 6.98(2 \mathrm{H}, \mathrm{s}), 6.63(2 \mathrm{H}, \mathrm{s}), 5.51(2 \mathrm{H}, \mathrm{dd}, J=2.2,7.1 \mathrm{~Hz}), 5.38$ (2H, dd, $J=1.9,8.7 \mathrm{~Hz}$ ), 4.15-4.09 (2H, br. m), 3.41-3.38 (2H, m), 3.10-3.05 (2H, m), 2.93-2.89 (2H, m), 2.72 (6H, s), 2.58-2.55 (4H, m), 2.40 (6H, br. m), 2.12 (6H, s), 2.11-2.07 (2H, m), 2.011.93 (2H, m), 1.80-1.54 (12H, m), 1.45-1.40 (2H, m), 1.38 (6H, s), 1.32-1.17 (2H, m), 1.13 (6H, s), $1.07(6 \mathrm{H}, \mathrm{d}, J=6.9 \mathrm{~Hz}), 0.94(6 \mathrm{H}, \mathrm{d}, J=6.8 \mathrm{~Hz}) ;{ }^{13} \mathrm{C} \mathrm{NMR}\left(100.6 \mathrm{MHz}, \mathrm{CDCl}_{3}\right) \delta 216.4(2 \mathrm{C})$, 172.8 (2C), 170.5 (2C), 165.5 (2C), 152.6 (2C), 120.5 (2C), 116.3 (2C), 78.3 (2C), 76.0 (2C), 73.9 (2C), 71.7 (2C), 57.4 (2C), 53.9 (2C), 52.4 (2C), 43.7 (2C), 38.4 (2C), 34.3 (2C), 33.9 (2C), 31.4 (2C), 29.6 (2C), 26.2 (2C), 24.9 (2C), 24.1 (2C), 22.2 (2C), 20.5 (2C), 19.1 (2C), 17.6 (2C), 15.7 (2C), 15.5 (2C); Anal. Calcd. for $\mathrm{C}_{58} \mathrm{H}_{84} \mathrm{~N}_{2} \mathrm{O}_{14} \mathrm{~S}_{2}$ : C, 63.48; H, 7.72; N, 2.55. Found: C, 63.51; H, 7.70; N, 2.51. $[\alpha]_{\mathrm{D}}^{25}=-4.3$ (c 0.36, $\left.\mathrm{CHCl}_{3}\right)$; HRESI positive MS: Anal. Calcd. for $\mathrm{C}_{58} \mathrm{H}_{84} \mathrm{~N}_{2} \mathrm{O}_{14} \mathrm{~S}_{2} \mathrm{Na}+, 1119.52562$; found 1119.52719 . 
$5:{ }^{1} \mathrm{H}$ NMR (400 MHz, $\left.\mathrm{CDCl}_{3}\right) \delta 6.98(2 \mathrm{H}, \mathrm{s}), 6.62(2 \mathrm{H}, \mathrm{s}), 5.50(2 \mathrm{H}, \mathrm{dd}, J=1.7,6.9 \mathrm{~Hz}), 5.37$ (2H, dd, $J=1.7,8.8 \mathrm{~Hz}$ ), 4.13-4.09 (2H, br. m), 3.39 (2H, dd, $J=6.9,8.6 \mathrm{~Hz}$ ), 3.10-3.07 (2H, m), 2.92-2.89 (2H, m), 2.72 (6H, s), 2.57-2.55 (4H, m), 2.35 (4H, t, J = $7.41 \mathrm{~Hz}), 2.14$ (6H, s), 2.122.08 (2H, m), 1.99-1.92 (2H, m), 1.75-1.53 (14H, m), 1.45-1.39 (6H, m), 1.38 (6H, s), 1.23-1.17 (2H, m), $1.13(6 \mathrm{H}, \mathrm{s}), 1.08(6 \mathrm{H}, \mathrm{d}, J=6.8 \mathrm{~Hz}), 0.94(6 \mathrm{H}, \mathrm{d}, J=6.8 \mathrm{~Hz}) ;{ }^{13} \mathrm{C}$ NMR $(100.6 \mathrm{MHz}$, $\mathrm{CDCl}_{3}$ ) $\delta 216.2$ (2C), 173.0 (2C), 170.4 (2C), 165.5 (2C), 152.3 (2C), 136.6 (2C), 120.1 (2C), 116.3 (2C), 78.1 (2C), 76.2 (2C), 73.9 (2C), 57.3 (2C), 53.9 (2C), 52.3 (2C), 43.8 (2C), 38.5 (2C), 34.5 (2C), 34.3 (2C), 31.5 (2C), 29.6 (2C), 28.8 (2C), 26.3 (2C), 24.8 (2C), 24.1 (2C), 22.1 (2C), 20.5 (2C), 19.0 (2C), 17.5 (2C), 15.5 (2C), 15.4 (2C); Anal. Calcd. for $\mathrm{C}_{60} \mathrm{H}_{88} \mathrm{~N}_{2} \mathrm{O}_{14} \mathrm{~S}_{2}$ : C, 64.03; H, 7.88; N, 2.55. Found: C, 64.06; H, 7.87; N, 2.51. MS-ESI (MeOH) m/z (\%) $1147.8(100)(M N a)^{+}$; $1126.0(26)(\mathrm{MH})^{+} ;[\alpha]_{\mathrm{D}}{ }^{25}=-6.8\left(\mathrm{c}=0.54, \mathrm{CHCl}_{3}\right)$; HRESI positive MS: Anal. Calcd. for $\mathrm{C}_{60} \mathrm{H}_{88} \mathrm{~N}_{2} \mathrm{O}_{14} \mathrm{~S}_{2} \mathrm{Na}+, 1147.55692$; found 1147.55527.

$6:{ }^{1} \mathrm{H}$ NMR (400 MHz, $\left.\mathrm{CDCl}_{3}\right) \delta 6.99$ (2H,s), 6.64 (2H,s), 5.52 (2H, br. d), 5.38 (2H, dd, $J=1.7$, 8.8 Hz), 4.14-4.09 (2H, br. m), 3.41-3.37 (2H, m), 3.10-3.06 (2H, m), 2.93-2.92 (2H, m), 2.73 (6H, s), 2.58-2.56 (4H, m), 2.35 (4H, t, $J=7.4 \mathrm{~Hz}), 2.15$ (6H, s), 2.10-2.08 (2H, m), 2.00-1.93 (2H, m), 1.75-1.54 (14H, m), 1.39 (6H, s), 1.36-1.18 (12H, m), 1.14 (6H, s), 1.08 (6H, d, J = 6.9 Hz), 0.95 (6H, d, $J=6.8 \mathrm{~Hz}){ }^{13} \mathrm{C}$ NMR (100.6 MHz, $\left.\mathrm{CDCl}_{3}\right) \delta 216.2$ (2C), 173.1 (2C), 170.4 (2C), 165.3 (2C), 153.7 (2C), 135.8 (2C), 120.1 (2C), 116.3 (2C), 78.4 (2C), 76.2 (2C), 73.9 (2C), 57.3 (2C), 53.9 (2C), 52.3 (2C), 43.9 (2C), 38.5 (2C), 34.5 (2C), 34.4 (2C), 31.8 (2C), 29.6 (2C), 29.4 (2C), 29.1 (2C), 26.3 (2C), 25.0 (2C), 24.6 (2C), 22.2 (2C), 20.5 (2C), 19.0 (2C), 17.5 (2C), 15.5 (2C), 15.4 (2C); Anal. Calcd. for $\mathrm{C}_{62} \mathrm{H}_{92} \mathrm{~N}_{2} \mathrm{O}_{14} \mathrm{~S}_{2}$ : C, 64.56; H, 8.04; N, 2.43. Found: C, 64.56; H, 8.04; N, 2.43. MS-ESI $(\mathrm{MeOH}) \mathrm{m} / \mathrm{z}(\%) 1176.7(100)(M N a)^{+}, 1154.0(72)(M H)^{+},[\alpha]_{\mathrm{D}}{ }^{25}=-3.5($ с 0.43 , $\mathrm{CHCl}_{3}$ ); HRESI positive MS: Anal. Calcd. for $\mathrm{C}_{62} \mathrm{H}_{92} \mathrm{~N}_{2} \mathrm{O}_{14} \mathrm{~S}_{2} \mathrm{Na}+, 1175.58822$; found 1175.58942 
Tubulin polymerization assay: Tubulin was isolated as pure $\alpha \beta$-tubulin (>95\%) from fresh pig brain according to the protocol previously reported. ${ }^{6}$ Tubulin was dissolved in BRB80 buffer (80 mM PIPES, $1 \mathrm{mM} \mathrm{MgCl}_{2}, 1 \mathrm{mM}$ EGTA adjusted to $\mathrm{pH} 6.8$ with $\mathrm{KOH}$ ), Sample was diluted in BRB80 buffer to a final volume of $1 \mathrm{~mL}$. Induction of polymerization of purified tubulin was determined in a centrifugation-based protein quantification assay. Compounds (5 $\mu \mathrm{M}$ and $10 \mu \mathrm{M}$ ) were incubated with tubulin $(5 \mu \mathrm{M})$ for $30 \mathrm{~min}$ at RT. The samples were subsequently centrifuged at $20000 \mathrm{~g}$ for $30 \mathrm{~min}$ at $4^{\circ} \mathrm{C}$ and the protein concentration determined in the supernatant by the Bradford method (Biorad, Switzerland). The level of polymer formation upon incubation of compounds with purified tubulin was compared to the amount of polymerized tubulin induced with $25 \mu \mathrm{M}$ Epo B, which gave maximal polymerization > 95\%. The \% tubulin polymerization values reported in Table 1 thus represent the ratio of polymer formation obtained with test compounds and $25 \mu \mathrm{M}$ Epo B, respectively. The \% tubulin polymerization was determined in 3 independent experiments.

Cell growth assay: $\underline{A 549}$ and $\underline{M C F-7} \mathrm{IC}_{50}$ values for human cancer cell growth inhibition were determined for a $72 \mathrm{~h}$ exposure period of cells to compounds by quantification of protein content of fixed cells by methylene blue staining (Meyer et al., 1989). For further experimental details, see Nicolau et al. (2000). The $\mathrm{EC}_{50}$ values given in the text and the $\mathrm{IC}_{50}$ values in the table all represent the means of three independent experiments ( \pm standard deviation); $\underline{H T 1080}$ : The 3-(4,5dimethylthiazol-2-yl)-2,5-diphenyltetrazolium bromide (MTT; Sigma Chemical Co., St. Louis, MO) dye reduction assay in 96-well microplates was used, as previously described (Rodríguez Nieto et al., 2001). $3 \times 10^{3}$ BAE or $2 \times 10^{3} \mathrm{HT} 1080$ in a total volume of $100 \mu \mathrm{L}$ of their respective growth media were incubated with serial dilutions of the tested compounds. After 3 days of incubation ( $37^{\circ} \mathrm{C}, 5 \% \mathrm{CO}_{2}$ in a humid atmosphere) $10 \mu \mathrm{l}$ of MTT (5 mg/ml in PBS) were added to 
each well and the plate was incubated for a further $4 \mathrm{~h}\left(37^{\circ} \mathrm{C}\right)$. The resulting formazan was dissolved in $150 \mu \mathrm{L}$ of $0.04 \mathrm{~N} \mathrm{HCl}-2$ propanol and read at $550 \mathrm{~nm}$. All determinations were carried out in triplicate. IC50 value was calculated as the concentration of compound yielding a $50 \%$ of cell survival. $\underline{\beta D 10}$ : Cells were harvested and plated in 96-well flat-bottomed microplates at a density of $10^{3}$ cells/well. Assays were performed in quintuplicates. Cells were allowed to attach for $24 \mathrm{~h}$. The drugs were prepared in medium at different concentrations and were added to the plates at a volume of $100 \mu \mathrm{l} /$ well. After 24 (or 96) h incubation $20 \mu \mathrm{l}$ of the CellTiter $96^{\circledR}$ AQueous One Solution Reagent (Promega Corporation, Madison, WI, USA) were added to each well and the plates were incubated for one hour at $37^{\circ} \mathrm{C}$. The CellTiter $96^{\circledR}$ AQueous One Solution Reagent contains a tetrazolium compound [3-(4,5-dimethylthiazol-2-yl)-5-(3-carboxymethoxyphe-nyl)-2-(4sulfophenyl)-2H-tetrazolium, inner salt; MTS] and an electron coupling reagent (phenazine ethosulfate; PES). PES has a high chemical stability, which allows it to form a stable solution with MTS. The absorbance was read at 490nm on a plate spectrophotometer (Victor ${ }^{3}$ тм 1420 Multilabel Counter, Perkin Elmer Instruments, Shelton, USA). Cell cytotoxicity was expressed as the percentage of the controls.

Endothelial cell differentiation assay: tube formation on Matrigel: Matrigel ( $50 \mu \mathrm{L}$ of about 10.5 $\mathrm{mg} / \mathrm{mL}$ ) at $4{ }^{\circ} \mathrm{C}$ was used to coat each well of a 96 -well plate and allowed to polymerize at $37^{\circ} \mathrm{C}$ for a minimum of 30 min as described previously. ${ }^{12}$ Some $5 \times 10^{4}$ BAE cells were added with $200 \mu \mathrm{L}$ of DMEM. Finally, different amounts of the tested compounds were added and incubated at $37^{\circ} \mathrm{C}$ in a humidified chamber with $5 \% \quad \mathrm{CO}_{2}$. After incubation for $7 \mathrm{~h}$, cultures were observed (200xmagnification) and photographed with a NIKON inverted microscope DIAPHOT-TMD (NIKON Corp., Tokyo, Japan). Each concentration was tested in duplicate, and two different observers evaluated the results of tube formation inhibition.

Endothelial cell migration assay: The migratory activity of BAE cells was assessed using a wound migration assay. ${ }^{13}$ Confluent monolayers in 6-well plates were wounded with pipette tips giving rise 
to one acellular $1 \mathrm{~mm}$-wide lane per well. After washing, cells were supplied with $1.5 \mathrm{~mL}$ complete medium in the absence (controls) or presence of the indicated concentrations of the tested compounds. Wounded areas were photographed at zero time and after 7, and $24 \mathrm{~h}$ of incubation, plates were observed under microscope and photos were taken from the same areas as those recorded at zero time.

\section{Acknowledgements}

This research has been developed under the umbrella of CM 0602 COST Action "Inhibitors of Angiogenesis: design, synthesis and biological exploitation". The authors are grateful to NOVARTIS for providing Epothilone A. D. Passarella and D. Comi are grateful to the financial support provided by Ministero dell'Istruzione, dell'Università e della Ricerca (MIUR) PRIN 2007 Program "Sviluppo e caratterizzazione di nuovi inibitori di tirosine chinasi cellulari con attività antiproliferativa e antiangiogenica nei confronti di differenti tumori”. A. R. Quesada is grateful to the financial support provided by Ministerio de Ciencia y Tecnología, Spain (CTQ2006-15279C03-03) and Junta de Andalucia (Proyecto de investigación de excelencia CTS-3759).

\section{References and Notes}

1. a) Nicolaou, K.C.; Roschangar, F.; Vourloumis, D. Angew. Chem. Ed. 1998, 37, 2014 - 2045; b) Attard, G.; Greystoke, A.; Kaye, S.; De Bono, S. Pathologie Biologie 2006, 54, 74 - 82.

2. a) Höfle, G.; Bedorf,N.; Gerth, K. and Reichenbach,H.; German Patent Disclosure, DE 4138042, 1993 (Priority Nov. 19, 1991), Chem. Abstr.,1993, 120, 52841; b) Gerth, K.; Bedorf, N.; Höfle, G.; Irschik, H.; Reichenbach,H. J. Antibiot., 1996, 49, 560 - 564

3. a) Lee, F. Y. F.; Borzilleri, R.; Fairchild, C. R.; Kim S.-H.; Long, B. H.; Reventos-Suarez, C.;

Vite, G. D.; Rose, W. C.; Kramer, R. A. Clinical Cancer Research , 2001, 7, 1429 - 1437; b) Cortes, J.; Baselga, J. The Oncologist. 2007, 12, 271 - 280; c) Rivera, E.; Lee, J.; Davies, A. The 
Oncologist. 2008, 13, 1207 - 1223; d) Kaminskas, E.; Jiang, X.; Aziz, R.; Bullock, J.; Kasliwal, R.; Harapanhalli,R.; Pope, S.; Sridhara, R.; Leighton, J.; Booth, B.; Dagher, R.; Justice, R.; Pazdur, R. Clin. Cancer Res. 2008, 14, 4378 - 4384; e) Hunt, J. T. Mol. Cancer. Ther., 2009, 8, 275 - 281

4. Thorpe, P. E.; Chaplin, D. J.; Blakey, D. C. Cancer Res. 2003, 63, 1144 - 1147.

5. Jain, R.K. Science 2005, 307, 58 - 62.

6. Gertsch, J.; Meyer, S; Müller, M; Altmann, K.-H. ChemBioChem 200910166 - 175

7. Nicolaou, K.C.; Hepworth, D.; King, N. P.; Finlay, M.R.V.; Scarpelli, R.; Pereira, M. M. A.; Bollbuck, B.; Bogot, A.; Werschkun, B.; Winsigger, N. Chem. Eur. J. 2000, 6, 2783 - 2800.

8. For other epothilones analogues see: a) Nicolaou, K. C.; Scarpelli, R.; Bollbuck, B.; Werschkun, B.; Pereira, M. M.; Wartmann, M.; Altmann, K.-H.; Zaharevitz, D.; Gussio, R.; Giannakakou, P. Chem. Biol. 2000, 7, 593 - 599; b) Watkins, E.B.; Chittiboyina, A.G.; Jung, J.C.; Avery, M.A. Curr. Pharm. Design 2005, 11, 1615 - 1653.

9. For other example of selective functionalization of C7-OH see: Sefkoe, M.; Kiffe, M.; Schummer, D.; Höfle, G. Bioorg, Med, Chem. Lett 1998, 8, 3025 - 3030.

10. Reamon-Buettner, S.M.; Borlak, J. Cancer Res. 2008, 68, 7587 - 7596.

11. Folkman, J. Nat. Med. 1995, 1, 27 - 31.

12. Rodríguez-Nieto, S.; Chavarría, T.; Martínez-Poveda B.; Sánchez-Jiménez, F.; Quesada, A. R.; Medina, M.A. Biochem. Biophys. Res. Commun 2002, 293, 497 - 500.

13. Martínez-Poveda, B.; Quesada, A. R.; Medina, M.A. Eur. J. Pharm. 2005, 516, 97 - 103. 